\title{
Near surface modification affected by hydrogen/metal interaction
}

\author{
Y. Katz, M. Tymiak \& W. W. Gerberich \\ University of Minnesota, Minneapolis, U.S.A
}

\begin{abstract}
Hydrogen/metal interaction represents a complex topic that attracted a high volume of experimental/theoretical efforts. Thus, for better understanding, ample activities have been conducted that spread over various levels and scales. The current study emphasizes some additional facets manifested mainly by confined and localized information. In this context, nano tests assisted by contact mechanics methodology provided small volume information to be even stretched to other surface related behaviour. This includes implications regarding the quantification of sequential events as related to hydrogen embrittlment (HE) or fracture processes transition. In addition, questions emerged regarding wear aspects or tribological contacts insights. Basically, the study considered free hydrogen (either external or internal) to be an aggressive environmental agent in elastic-plastic crystalline solids. Experimentally based information has been gathered in the bulk, supplemented also on the nano scale. In fact, this comprehensive study with emphasis on the scale effects provided striking insights. For example, in terms of contact tribological effects, normally mechanical driving force is solely considered. However, here, the unique contribution by environmental interaction includes phase stability effects and localized plasticity. Mainly two materials have been focused: first, as a background, AISI 316L metastable austenitic stainless steel, and secondly $\mathrm{Ti} / \mathrm{Cu}$, thin film specimens affected by hydrogen. The outcoming effects have been sorted out by mechanical response tracking and morphological visualization. Quantitatively, it was assessed by nano indentation and continuous scratch tests. Besides mechanical testing the information was supplemented by scanning Probe Microscopy (SPM) observations. It became evident that hydrogen affected dramatically the investigated systems with exploration of dislocation nucleation and dynamics. As such, remarkable differences occurred on both plastic localization and micro crack onset. In the thin layers the effective work of adhesion was reduced, indicating significant degradation that could be expressed quantitatively. Finally it was concluded that since fracture is a localized phenomena nano scale information might suggest "critical experiments" so vital for fundamental concepts confirmation.

Keywords: hydrogen embrittlment, metastable austenitic stainless steel, nano data, dislocation emission, wear.
\end{abstract}




\section{Introduction}

Hydrogen/metal interactive effects have significant implications on structure integrity aspects due to crack stability transition. Regardless the specific enhancing damage origins, crack nucleation and growth are critical forms of mechanical degradation events. Note that susceptibility to aggressive environment requires special concern regardless the specific structural scale. Attention to small volume circumstances initiated alternative avenues in order to expand the spectrum of insights into hydrogen/deformation interactive effects. The current study selected mainly metastable austenitic stainless steel as well as $\mathrm{Ti} / \mathrm{Cu}$ thin layers system. Previous studies established already that phase stability aspects in austenitic stainless steel are either enhanced by solely mechanical driving force or by hydrogen interaction. Hydrogen can be provided externally, internally or both. For the sake of the current investigation, beside hydrogen availability due to residuals at microstructural trapping sites, hydrogen was charged intentionally. The charging process can be achieved by electrolytic cathodic charging or by high temperature/pressure gasous charging. Based on previous experimental confirmation it was concluded that regardless the exact procedure of hydrogen charging, austenite decomposition in austenitic stainless steel prevails [1,2]. However, major differences are more associated to the damage evolution caused by a more aggressive high fugacity charging $[3,4]$.

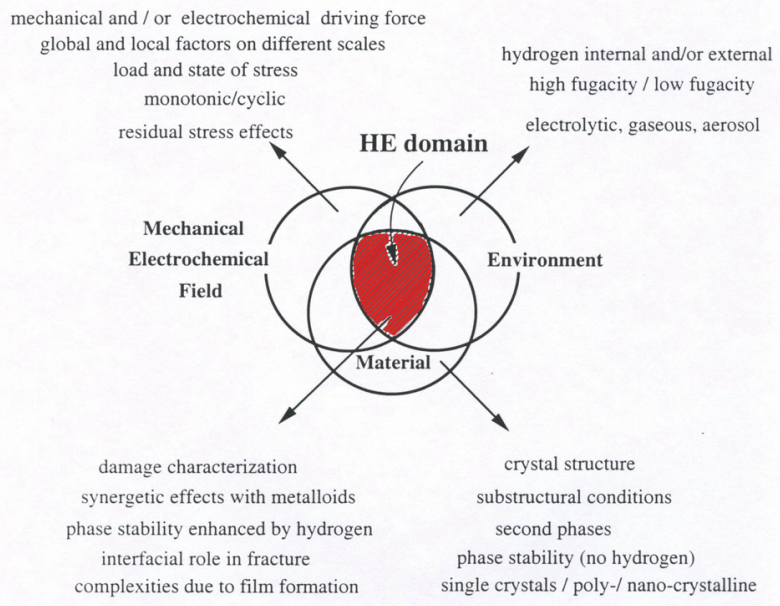

Figure 1: Global description of hydrogen/Metal interaction aspects.

Generally, the following notion remains in order by emphasizing that the present study is engaged only with free hydrogen effects, in contrast to the role of hydride formation that provides other damaging origins. In order to 
summarize the intense efforts that have been invested in the understanding of the highly complex hydrogen/deformation interactions, some of the variables that dominate this interactive process are depicted in Fig. 1. In the current study, the two selected materials that seem to be hardly related represent at least the small volume approach. The distinction between highly localized resolutions compared to macroscopic contact mechanics damage model, expanded the interpretation capabilities. For example, friction and wear damage evolution have been treated macroscopically from a cumulative damage standpoint $[5,6]$. In contrast the nano data provided information that enabled to deal also with point contact (asperities). Moreover, yield excursions alluded to dislocation emission activity and microplasticity analysis. Since the study proposes practical measurements potential, the initial stages of wear - damage might be tackled. The metastable stainless steel is affected by free hydrogen in a typical surface upset beside crystal plasticity habits, phase transformation and micro cracks formation. The thin films emphasize the interfacial strength variability or delamination that clearly affects tribological contact behaviour. These were manifested by ultra fine features that could be measured at the near surface.

\section{Experimental procedures}

In the bulk surfaces of 304L, 316L and 310 metastable stainless steels, thermal stability was previously established. In this context, even after the immersion into liquid nitrogen and helium the monolithic austenitic phase $(\gamma)$ was preserved in all three materials. Similar results were obtained after plastic straining at ambient temperature. However, in the 304L stainless steel after very extensive straining at $296 \mathrm{~K}$ small amount of the body centered austenitic phase was detected. Completely different behavior occurred under load at cryogenic temperatures and straining at $77 \mathrm{~K}$ in which significant austenite decomposition occurred. The transformation characterization has been assisted by X-ray diffraction and Mossbauer transmission spectra with emphasis on the composition and the reaction sequence. For the latter, the reaction was confirmed to be $\gamma \rightarrow \varepsilon^{\prime}+\alpha^{\prime}$ where $\varepsilon^{\prime}$ and $\alpha^{\prime}$ are the hexagonal close packed and the body centered tetragonal martensitic phases respectively [7]. For example, X-ray diffraction enabled to detect the intermediate $\varepsilon^{\prime}$ hcp martensitic phase at the early stage of 0.02 true strain. More about the austenitic stainless steels have been addressed elsewhere $[3,4]$. Here to mention that the $316 \mathrm{~L}$ stainless steel behaved similarly to the 304 besides revealing always higher thermo-mechanical stability. The exceptional stability behavior of the 310 stainless steel became apparent after extensive straining at $77 \mathrm{~K}$. Once again, phase stability degree was a major distinction between the aforementioned austenitic steel types. In contrast to hydrogen-free systems, the case of hydrogen interaction highly depends on the hydrogen charging namely the fugacity degree conditions. Specimens were prepared from flat materials by utilizing spark erosion for the metal working technique; followed by electrolytic polishing. By using such procedures cold working influences were drastically reduced. In most of the specimen series, hydrogen was introduced at ambient temperature by cathodic charging from 1 to 
16 hours at a current density of $50-500 \mathrm{~mA} / \mathrm{cm}^{2}$. The electrolytic cell consisted of platinum coil as the anode in $1 \mathrm{~N}_{2} \mathrm{SO}_{4}$ aqueous solution with $250 \mathrm{mg}$ arsenite per one liter. The hydrogenated specimens were examined periodically for their structure stability confined to the affected hydrogen interfaced layer. The X-ray diffractometry was performed by using shallow penetrating $\mathrm{CuK}_{\alpha}$ radiation, utilizing also a graphite-monochromator that eliminated the high fluorescence background radiation. For resolving the hydrogen affected layer deeper penetrating radiation like $\mathrm{MoK}_{\alpha}$ or $\mathrm{CoK}_{\alpha}$ were supplemented. For the Mossbauer transmission spectroscopy, a constant acceleration computerized spectrometer was used in conjunction with a $25 \mathrm{mCi}^{57} \mathrm{Co}(\mathrm{Pd})$ source. For this purpose, hydrogen-free specimens were electrolytically thinned to about $25 \mu \mathrm{m}$ while for hydrogen effect studies it was necessary to reduce the specimen thickness to $5 \mu \mathrm{m}$ prior to hydrogen charging. Also here, specimens were prepared by following the aforementioned procedures namely, by utilizing spark erosion for metal working process, followed by electrolytic polishing. The X-ray diffraction and the Mossbauer spectra analysis intended to engage with the following factors. The lattice expansion and contraction, the role of preferred orientation on magnetic polarization, overlapping of the (hkl) reflection peaks, the hydrogen affected layer size and products concentration. Beside others, complementary studies were conducted, i.e., gas chromatography and hydrogen collection measurements combined with microscopic gas release analysis. Here to mention that the quantitative analysis considered also the Debye-Waller factor influences. In addition to the bulk surfaces information, nano scale procedures have been conducted with attention to hydrogen/stainless steel interaction. For this purpose, the selected material was AISI 316L metastable stainless steel a polycrystalline systems that consisted of $50-100 \mu \mathrm{m}$ grain size. Mechanical response and plasticity behaviour with and with no hydrogen was characterized by contact mechanic methodology including mainly nano indentation and lateral nano scratch techniques. In this case hydrogen was charged also by cathodic charging namely by $1 \mathrm{M} \mathrm{NaOH}$ under current densities in the range of 10 to 500 $\mathrm{mA} / \mathrm{cm}^{2}$. Similar to the previous post charging studies, sequential events vs. elapsed times were tracked during hydrogen out gaseous. For the nano indentation test, the mentioned microstructure grain size enabled at least ten consecutive tests to be conducted within the same grain. Fine features visualization was carried out for the nano indentation as well as for the scratch trances. These functions were performed by Scanning Electron Microscopy (SEM) and by Atomic Force Microscopy (AFM).

\section{Experimental results}

\subsection{The metastable stainless steel system: global approach}

Second phase aspects in austenitic stainless steels considered the austenitic decomposition enhanced by mechanical, hydrogen or both interactions. In fact this whole material class is unstable below the Md temperature even with no hydrogen. Moreover, hydrogen with or with no mechanical field can result in 
martensitic transformation, mechanical degradation due to localized displacement, delayed cracking and ductility reduction. In this context, few phenomenological observations are cited involving mainly electrolytic charging. Studies in 304, 316 and 310 stainless steel have covered a relatively wide range of phase stability as related to the degree of mechanical degradation. For example, high fugacity charging of 304L with an initial concentration of 0.34 $\mathrm{H} / \mathrm{M}$ at. resulted in an affected layer of about $1 \mu \mathrm{m}$. With no mechanical field, about 0.3 volume fractions of transformed austenitic products were observed in contrast to 0.85 volume fraction with an imposed true strain of 0.28 . Generally (except for the 310 case) the early work of Holzworth and Louthan [1] indicated that similar phases resulted from plastically strained material compared to those charged with hydrogen. It is emphasized that phase transformation and the related fracture modes have been established regardless a specific charging procedure methods (Fig. 2). The transformation reaction pattern that was already mentioned takes a different form during the transient time, in which expanded $\varepsilon^{*}$ and $\alpha^{*}$ were confirmed. These were identified as pseudo-phases by X-ray diffraction and internal friction studies [8].

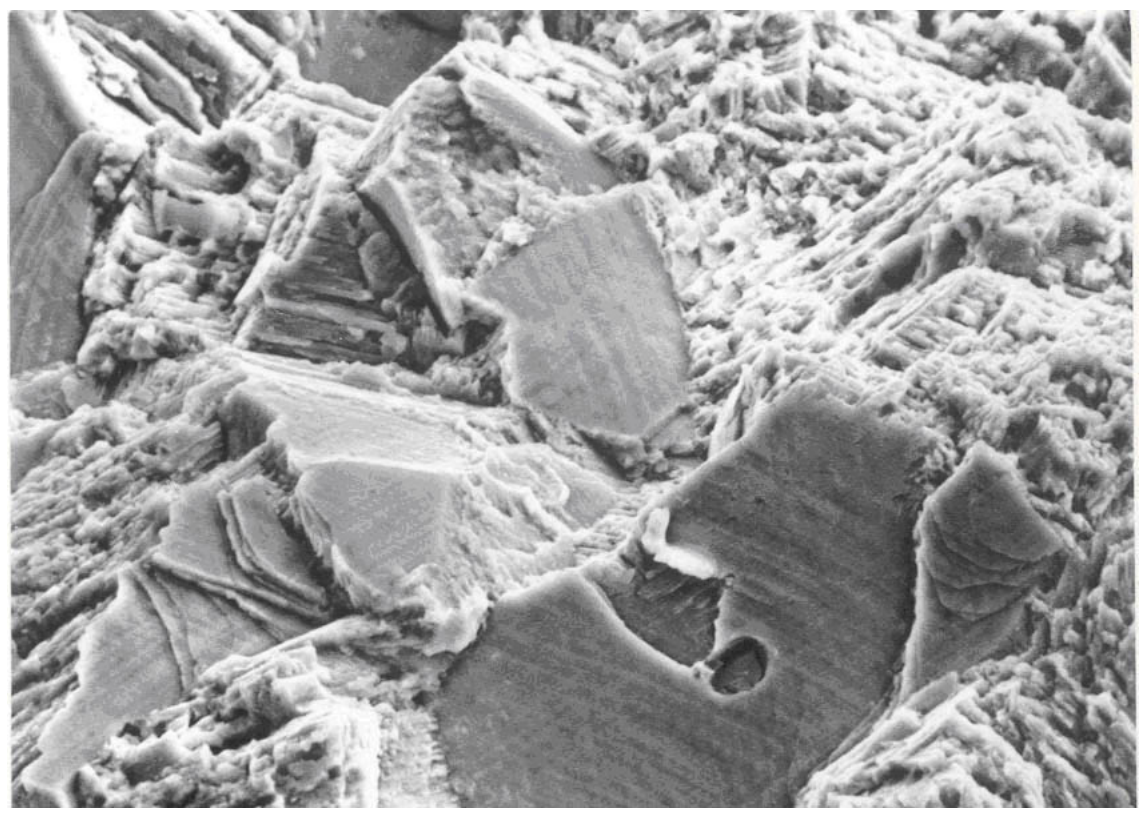

Figure 2: $\quad$ SEM fractograph indicating alternative modes in hydrogenated 316L stainless steel.

\subsection{Metastable 316L stainless steel system: local approach}

Indentation tests for a prescribed load of $1000 \mu N$ were carried out with Hysitron nano indentation device utilizing a $90^{\circ}$ conical indenter with a $400 \mathrm{~nm}$ tip radius of curvature. Tests were performed prior to hydrogen charging, instantly post- 
charging and one day after charging. Reproducible displacement excursions at an average load of $200 \mu N$ were observed for the non-charged samples as show in Fig. 3.

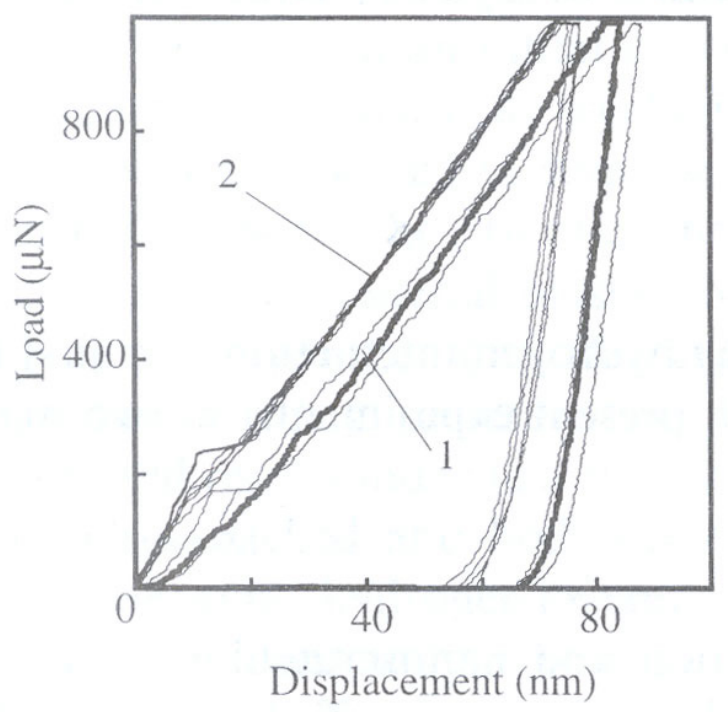

Figure 3: Indentation into non-charged samples. 1 and 2 correspond to arrays of indents in two different areas.

These were attributed to plasticity initiation since unloading just before the excursion load yielded no residual deformation. One example of hydrogen charged specimen is illustrated in the load displacement curve (Fig. 4). Here yield initiation occurred at $650-700 \mu N$. One day after charging the yield points ranged between 300-350 $\mu N$. An interesting observation supporting hydrogen charging induced impediment of dislocation motion is that, the load-depth dependence after yield initiation appears to be different from charged samples as compared to non-charged ones. Regarding the scratch test, it appeared that hydrogen increased localized plasticity along a given slip band by as much as a factor of three. In principal, quantitative local strain $(\lambda)$ arguments based on the surface slip height (h) and spacing (s) could be developed. Similar to what has been estimated as cumulative strain damage in fatigue such measurements of localized slips could be accomplished also here. These in fact are the first estimates of localized slip by probe microscopy as enhanced by hydrogen uptake. Thus, the following can be summarized at this stage. Surface modification due to environmental interaction in metastable austenitic stainless steel result at least from two origins.

First, strain due to phase stability aspects and secondly from hydrogen enhance localized plasticity that could be measured. Clearly, results like microcracking or other damaged sites introduce additional wear variations. 


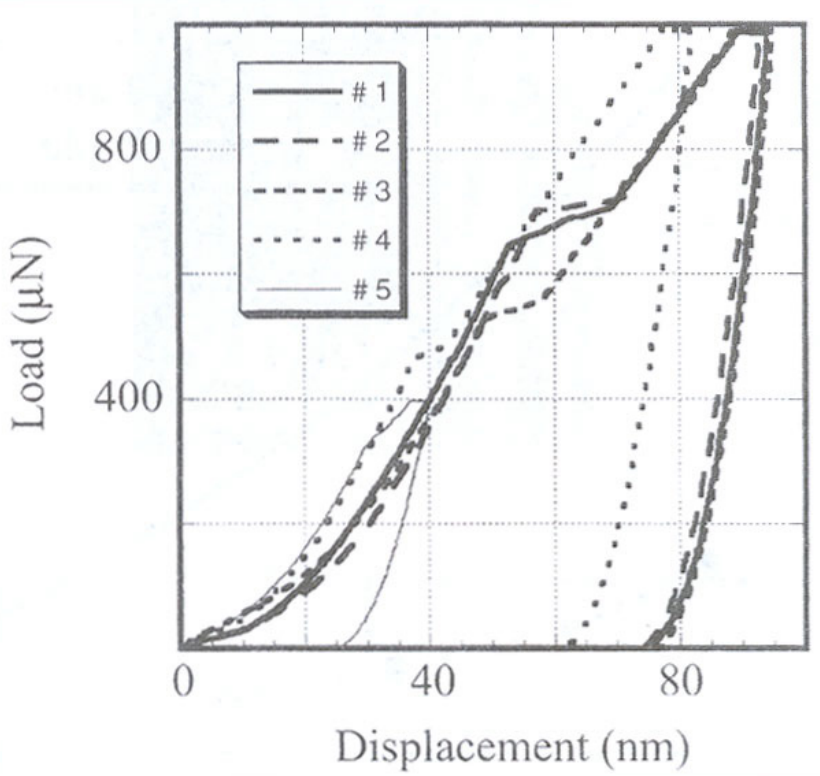

Figure 4: Nano indentation instantly after charging at high hydrogen concentration, time increases from 1 to 4 over $35 \mathrm{~min}$ time frame.

\section{Discussion and conclusion}

In this paper the main attention remains in exploring the near surface modification affected by hydrogen/metal interaction. Even so, the connection to hydrogen embrittlment phenomenon activities becomes understandable. Hydrogen interaction often forms a near surface affected layer caused by sequential events that are manifested from localized irreversible displacements up to micro cracking onset. Microscopic observations have revealed consistently that austenite decomposition enhanced also by hydrogen beside mechanical driving force is accompanied by the formation of a characteristic surface relief. In this context, bands in certain crystallographic directions were developed shortly after hydrogen charging. At this stage bands were observed with faint appearance gradually becoming clearer with elapsing time. Further examination under polarized light has revealed the anisotropic nature of the aforementioned bands network. The bands size enabled to evaluate the magnitude of the hydrogen affected layer thickness in the order of $1.5 \mu \mathrm{m}$. For specific hydrogen charging conditions displacements on one hand and micro cracking onset become important findings in terms of the surface characterization. On the ultra refined scale, two sources of information become quantitatively striking and could be briefly summarized. The nano indentation revealed major changes in the band behavior after hydrogen charging. In this case of 316L stainless steel, after low fugacity hydrogen charging, the initial yield increase is related to the 
increased hydrogen and its resultant stress gradient. It is still to be determined what the relative magnitude of the residual stress effect is compared to the hydrogen-dislocation interaction at the onset of plasticity. From the other source of the fine features observation namely the scratch test, it appeared that hydrogen increased the localized plasticity along a given slip band by as much as a factor of three. In principal, surface modification is based here on quantitative local plasticity arguments founded on measured localized strain. This was established by measured values of the surface slip height (h) and the slip spacing (s) as given in Table 1.

Table 1: $\quad$ Scratch test findings on plastic deformation.

\begin{tabular}{|c|c|c|c|}
\hline \multicolumn{2}{|c|}{ Surface features } & Non-charged & charged \\
\hline \multirow{2}{*}{ Along the pile-up } & $\begin{array}{c}\text { Slip step spacing } \\
(\mathrm{nm})(\mathrm{s})\end{array}$ & 101 & 291 \\
\cline { 2 - 4 } & $\begin{array}{c}\text { Slip step height } \\
(\mathrm{nm})(\mathrm{h})\end{array}$ & 15 & 94 \\
\hline \multirow{2}{*}{$\begin{array}{c}\text { Perpendicular to the } \\
\text { pile-up }\end{array}$} & $\begin{array}{c}\text { Slip step spacing } \\
(\mathrm{nm})(\mathrm{s})\end{array}$ & 106 & 261 \\
\cline { 2 - 4 } & $\begin{array}{c}\text { Slip step height } \\
(\mathrm{nm})(\mathrm{h})\end{array}$ & 13 & 16 \\
\hline
\end{tabular}

This in fact is the first estimate of the localized strain $(\lambda \sim \mathrm{h} / \mathrm{s})$ assisted by micro probe capabilities, providing surface characterization that was modified by hydrogen uptake. By utilizing nano indentation a significant contribution can be achieved not only regarding the localized plasticity behavior but also to the excursions as related to dislocations dynamics bounded to the operating slip systems. The described surface modification clearly has connectivity to the mechanisms affecting the local friction and the wear of tribological contacts. Moreover, some of the experimental procedures alluded to new approaches to be applied in exploring asperity contact effects in an initial stage of wear. For example, Kubota et al [9] addressed the issue of fretting fatigue systems concluding the significant life decrease caused by hydrogen interaction. Accordingly the following is summarized and concluded:

Critical experiments might eventually provide the building blocks for modeling effort in order to simulate hydrogen/metal interaction. It becomes apparent that novel techniques regarding localized contact mechanics and fracture processes can synergistically evaluate modified surfaces caused by complex interaction. Thus:

(1) Macro and micromechanical testing of hydrogen/deformation interaction suggest mutual supplementary information as related to multi-scale modeling development. 
(2) Hydrogen enhances near surface modification with implications to structural integrity aspects.

(3) Sorting out the origins for surface modification due to environmental interaction become possible.

(4) Applications that include in service segments in contact depend highly on surface characterization. An implication to fretting fatigue is only a selected example.

\section{References}

[1] Holzworth, M.I., \& Louthan, M.R., J.Corrosion-NACE, 24, pp110-124, 1968.

[2] Mathias, H., Katz, Y., Nadiv. S., Sec Int. Cong' on hydrogen in metals. 2C11,Paris,pp6-11,1977.

[3] Gilad, I. \& Katz. Y., Zeiteschrift fur physikalisch chemie, Genfold Bd, $1645,1989$.

[4] Mathias, H., Katz, Y., Nadiv. S., Metal hydrogen sys, Veziroglu, T.N., (ed), Oxford pergamon press, pp 225-249, 1982.

[5] Lim, S.C., \& Ashby, M.F., Acta metall, 35,1,1987.

[6] Blau, P., Friction and wear transition of materials, Park Ridge, NJ, Noyes, 1989.

[7] Mathias, H., Katz, Y., Nadiv. S., Metal Sci, 12, pp 129-137,1978.

[8] Gavrilijuk, V.G., Hanninen, H., Tarasenko, A.V., Terechenko, A.S., \& Ullakko, K., Acta metall mater, 43,559,1995.

[9] Kubota, M., Noyama, N., Fuata, N., Sakae, C., \& Kondo, Y., J. of the soc. of mater. Sci., Japan, 54, pp1231-1236, 2005. 